\title{
The prevalence of type 2 diabetes in people with psychiatric disorders: an umbrella review protocol
}

\author{
N. Lindekilde $1^{*}$, S. Scheuer ${ }^{2}$, F. Rutters ${ }^{3}$, J. E. Henriksen ${ }^{4}$, K. H. Rubin ${ }^{5}$, M. Lasgaard ${ }^{6}$, G. S. Andersen ${ }^{2}$ and \\ F. Pouwer ${ }^{1,4,7}$
}

\begin{abstract}
Background: Many epidemiological studies have investigated the prevalence of type 2 diabetes in individuals with a psychiatric disorder. In an umbrella review, we aim to systematically summarize existing systematic reviews examining the prevalence of type 2 diabetes in people with a psychiatric disorder. When information is available in the identified systematic reviews, comparisons with control groups without a psychiatric disorder will be made. Furthermore, we aim to assess the quality of the included systematic reviews.

Methods: The umbrella review will be based on a comprehensive systematic search of systematic reviews of observational (cross-sectional or longitudinal) studies investigating the prevalence of type 2 diabetes in people with a psychiatric disorder. Four electronic databases (Embase, PsycINFO, PubMed, and the Cochrane Database of Systematic Reviews) will be searched. Retrieved papers will be screened for eligibility by two independent reviewers. Furthermore, the reference lists of all included publications will be screened. Data will be extracted by using an a priori developed data extraction form and two independent reviewers will assess the risk of bias in the included systematic reviews using with the Risk of Bias in Systematic Reviews (ROBIS) tool. A narrative data-synthesis and a subsequent meta-analysis based on the primary studies will be made.
\end{abstract}

Discussion: For each psychiatric disorder, the data regarding the prevalence of type 2 diabetes will be summarized and discussed. When possible, comparisons with control groups will be reported and discussed. Finally, future implications and recommendations for clinical care will be presented.

Systematic review registration: This protocol was submitted for registration with the International Prospective Register of Systematic Reviews (PROSPERO) on December 9, 2019 (registration number: pending).

Keywords: Type 2 diabetes, Psychiatric disorder, Prevalence, Umbrella review, Systematic review

\section{Background}

Psychiatric disorders are common conditions. According to a systematic review from 2014, almost 1 in 5 adults worldwide meet the criteria for a psychiatric disorder during a 12-month period [1]. The life-time prevalence of psychiatric

\footnotetext{
* Correspondence: nlindekilde@health.sdu.dk

'Department of Psychology, University of Southern Denmark, Campusvej 55, 5230 Odense M, Denmark

Full list of author information is available at the end of the article
}

disorders was 29\% (26-33\%) [1]. Different psychiatric disorders do not only seriously impact the quality of life of those affected and their family members, the disorders may also have a negative impact on other health outcomes. For example, a systematic review of longitudinal studies showed that mortality rates were significantly higher in people with psychiatric disorders than in those without such disorders (pooled relative risk $=2.22 ; 95 \% \mathrm{CI}, 2.12-2.33 ; n=133$

(c) The Author(s). 2020 Open Access This article is licensed under a Creative Commons Attribution 4.0 International License, which permits use, sharing, adaptation, distribution and reproduction in any medium or format, as long as you give appropriate credit to the original author(s) and the source, provide a link to the Creative Commons licence, and indicate if changes were made. The images or other third party material in this article are included in the article's Creative Commons licence, unless indicated otherwise in a credit line to the material. If material is not included in the article's Creative Commons licence and your intended use is not permitted by statutory regulation or exceeds the permitted use, you will need to obtain permission directly from the copyright holder. To view a copy of this licence, visit http://creativecommons.org/licenses/by/4.0/. The Creative Commons Public Domain Dedication waiver (http://creativecommons.org/publicdomain/zero/1.0/) applies to the data made available in this article, unless otherwise stated in a credit line to the data. 
studies) [2]. Furthermore, the median years of potential life lost was 10 years ( $n=24$ studies) [2].

It is important to better understand how different psychiatric disorders can impact long-term health outcomes. One adverse outcome is diabetes, which is a chronic metabolic condition that affected approximately 463 million people worldwide in 2019 [3]. Ninety percent of all people with diabetes are diagnosed with type 2 diabetes [4]. Many studies have shown a link between psychiatric disorders and type 2 diabetes [5-7]. In the last decades, complex associations between psychiatric disorders and type 2 diabetes have been theorized and investigated. For example, in 2014, de Jonge et al. determined the association in a large crossnational study that used diagnostic psychiatric interviews and data on self-reported diabetes. In this study, after adjusting for co-morbid psychiatric disorders, particularly depression, intermittent explosive disorder, binge eating disorder, and bulimia nervosa were associated with diabetes [5]. For several psychiatric disorders, it has been postulated that they have a bidirectional association with type 2 diabetes [6, 7]. Already 10 years ago, De Hert et al. described diabetes and cardiovascular diseases as significant issues in patients with severe mental illness [8]. However, the care for diabetes in people with psychiatric disorders remains far from optimal [9]. A recent Danish study indicated lower quality of diabetes care in people with diabetes and schizophrenia, compared to people with diabetes, but without schizophrenia (relative risk $[R R]=0.91,95 \%$ confidence interval $[\mathrm{CI}]=0.88-0.95)[10]$. For example, people with schizophrenia were less likely to receive foot and eye examinations $(\mathrm{RR}=0.96, \mathrm{CI}=0.93-0.99 ; \mathrm{RR}=0.97, \mathrm{CI}=$ 0.94-0.99), blood pressure monitoring $(\mathrm{RR}=0.98, \mathrm{CI}=$ 0.96-0.99), and treatment with antihypertensive drugs $(\mathrm{RR}=0.83, \mathrm{CI}=0.70-0.97)$ [10]. If a relatively large number of people with psychiatric disorders also have type 2 diabetes, this may put already vulnerable populations at increased risk for future health problems such as macroand microvascular diseases.

Several systematic reviews have explored the prevalence of type 2 diabetes in people with different psychiatric disorders such as schizophrenia, major depression, bipolar disorder, and post-traumatic stress disorder (PTSD), indicating that the prevalence of type 2 diabetes is higher in people with psychiatric disorders, compared to people without psychiatric disorder [11-14]. To date, in a meta-analysis of 25 studies, Stubb et al. found that 9.5\% of people with schizophrenia had type 2 diabetes and pooled relative risk compared to healthy control was 1.82 (95\% CI 1.56-2.13, $n=4,489,125)$ [11]. Similarly, Vancampfort et al. estimated the prevalence of type 2 diabetes in people with major depression disorder, bipolar disorder, or PTSD to be $8.7-10 \%$ [12-14].

However, a comprehensive and systematic umbrella review with a critical appraisal of the existing literature into the prevalence of type 2 diabetes in people with different psychiatric disorders is lacking. Such an umbrella review of existing systematic reviews will not only help clarifying whether there are differences in the prevalence of type 2 diabetes in people with different psychiatric disorders, but will also contribute to evaluating the quality of the existing evidence.

\section{Objective}

The umbrella review that we have planned to conduct aims to systematically summarize existing literature systematic reviews describing the prevalence of type 2 diabetes in people with a psychiatric disorder such as major depression, schizophrenia, PTSD, bipolar disorder, sleep disorder, or an anxiety disorder. Furthermore, when information is available in the identified systematic reviews, comparisons with control groups (from the general population or control groups without a psychiatric disorder) will be made. Finally, an assessment of the quality of the included systematic reviews will be conducted and discussed, providing directions for future research.

\section{Methods}

This protocol is developed in accordance with PRISMAP [Preferred Reporting Items for Systematic Reviews and Meta-Analyses Protocols] guidelines [15] (see Additional file 1). In accordance with the guidelines, the umbrella review is registered in PROSPERO (International Prospective Register of Systematic Reviews; registration number: ) in which any important protocol amendments will be noted.

\section{Search strategy}

We plan to search in four electronic databases: Embase (1974 to present), PsycINFO (1967 to present), PubMed (1966 to present), and Cochrane Database of Systematic Reviews (1992 to present). Furthermore, reference lists from included papers will be manually screened for further eligible papers.

Four domains will be used in the following search: (I) psychiatric disorder, (II) prevalence, (III) diabetes, and (IV) systematic review. The search terms that capture the different psychiatric disorders will be taken from the DSM-III, DSM-IV-TR (axis I and axis II) $[16,17]$, DSM5 (section II) [18], and the ICD-10 Classification of mental and behavioral disorders (F00-F99) [19]. The four domains will be included in a four-step search combining relevant search words and $\mathrm{MeSH}$ terms with Boolean Logic operators (OR and AND). Search terms within each domain are combined with the operator "OR" and the different domains are combined with the operator "AND." The search strategy has initially been targeted PubMed 
and will subsequently be adapted for the other databases. Additional file 2 shows the complete search string that will be used.

\section{Eligibility criteria}

In the umbrella review, we will include systematic reviews focusing on cross-sectional and longitudinal observational studies in humans. The following eligibility criteria will be used:

i. A systematic review (with or without a metaanalysis), describing a systematic search string and eligibility criteria. Posters and abstracts describing a systematic review are not included.

ii. Focus on an adult population ( $\geq 18$ years) with one or more psychiatric disorders (measured by, e.g., diagnostic interviews, hospital records, medical prescription, or self-reported measures). Psychiatric disorders include psychiatric diagnoses mentioned in the ICD or DSM classifications or elevated levels of clusters of psychiatric symptoms (DSM-III or DSM-IV, axis I or axis II; DSM5 section II; ICD-10, F00-F99). Studies focusing on psychotropic medication that describe the prevalence of type 2 diabetes are also included. Studies that focus on a single psychiatric symptom and/or distress not described in the ICD or DSM classifications will not be included in the umbrella review (e.g., work-related stress and short sleep duration).

iii. Measure of prevalence of type 2 diabetes (measured by, e.g., diagnosis, medical reports, medical prescription, or self-reported measurement). If no information is available regarding subtypes of diabetes, we expect that the majority will have type 2 diabetes and include these studies.

Only publications in English, a Scandinavian language, German, or Dutch will be included. No exclusion criteria related to publication date will be set.

After removing duplicates, two independent reviewers will screen titles and abstracts to identity potential eligible papers. If at least one of the reviewers qualifies the systematic review as potentially eligible after reading the abstract, the full text will be retrieved and assessed according to the eligibility criteria. If there are any disagreements after the full-text screening, the two reviewers will discuss until consensus is reached. If necessary, a third reviewer will be involved in the discussions. Reference lists of the included publications will be manually screened with the aim to identify additional eligible publications. A PRISMA flowchart will describe the selection process. The screening of studies will be handled in EndNote and Covidence [20].

\section{Data extraction and data-synthesis}

The data from the included systematic reviews will be extracted using a data extraction form that has been developed a priori. From each of the eligible systematic reviews the following information will be extracted: first author, year of publication, country, type of study, type of assessment of psychiatric disorder(s), type of assessment of type 2 diabetes, the number of studies included in the systematic review, total number of participants, and primary findings, including information on the prevalence of type 2 diabetes, odds ratios, and relative risk ratios, when possible. Furthermore, the number of cases and controls in each of the primary studies included in systematic reviews will be extracted. If the number of participants in the primary studies is not reported in the systematic reviews, the information will be retrieved from the papers describing these primary studies.

All systematic reviews will be summarized, and a narrative data-synthesis will be performed and presented for each of the psychiatric disorders identified in the umbrella review. Additionally, to summarize the reported prevalence of type 2 diabetes, we plan to conduct a meta-analysis of prevalence (a random effects model and 95\% confidence interval) for each of the psychiatric disorders [21]. Our meta-analyses will be based on information from the primary studies included in the systematic reviews to make sure that the primary studies will only be included once [22]. We plan to assess between-study heterogeneity (quantified as $I^{2}$ metric) $[23,24]$ and publication bias (small study effects, Egger's test) [25].

\section{Risk of bias assessment}

The strength of an umbrella review depends on the quality of the included systematic reviews. Therefore, it is important to assess the risk of bias in the included systematic reviews. Risk of bias assessment will be performed with the Risk of Bias in Systematic Reviews (ROBIS) tool [26].

\section{Discussion}

The evidence from systematic reviews regarding the prevalence of type 2 diabetes in people with psychiatric disorders will be summarized. Furthermore, potential prevalence differences between people with psychiatric disorders and control groups without psychiatric disorders will be discussed and the implications for clinical care will be presented. When relevant, differences in assessment methodology will be discussed. If meaningful, the potential mechanisms explaining the prevalence of type 2 diabetes among individuals with a psychiatric disorder will be hypothesized and discussed. The quality of the included systematic reviews will be discussed, and implications presented. Finally, the results will most 
likely also be used to provide recommendations for future research.

\section{Supplementary information}

Supplementary information accompanies this paper at https://doi.org/10. 1186/s13643-020-01341-6.

Additional file 1. PRISMA-P checklist.

Additional file 2. Search strategy.

\section{Abbreviations}

PRISMA-P: Preferred Reporting Items for Systematic Reviews and MetaAnalyses Protocols; RR: Relative risk

\section{Acknowledgements}

Not applicable.

\section{Authors' contributions}

All authors have contributed in a meaningful way. NL and FP had the original idea and NL made the first draft of the protocol. All authors (NL, SS, $F R$, JEH, KHR, ML, GSA, and FP) have commented on the protocol and likewise, all authors have read and approved the final manuscript.

\section{Funding}

The faculty of Health Sciences, University of Southern Denmark, has supported NL and her work on the present protocol and the umbrella review. The financial provider is not involved in project.

\section{Availability of data and material}

Not applicable.

\section{Ethics approval and consent to participate}

Not applicable.

\section{Consent for publication}

Not applicable.

\section{Competing interests}

G.S.A. own shares in Novo Nordisk A/S. The rest of the authors declared that they have no competing interests.

\section{Author details}

'Department of Psychology, University of Southern Denmark, Campusvej 55, 5230 Odense M, Denmark. ${ }^{2}$ Clinical Epidemiology, STENO Diabetes Center Copenhagen, Gentofte, Denmark. ${ }^{3}$ Department of Epidemiology and Biostatistics, Amsterdam Public Health Institute, Amsterdam UMC, location VUMC, Amsterdam, The Netherlands. ${ }^{4}$ STENO Diabetes Center Odense, Odense University Hospital, Odense, Denmark. ${ }^{5}$ OPEN - Open Patient data Explorative Network, Department of Clinical Research, University of Southern Denmark and Odense University Hospital, Odense, Denmark. ${ }^{6}$ DEFACTUM Public Health \& Health Services Research, Central Denmark Region, Aarhus, Denmark. 'School of Psychology, Deakin University, Geelong, Australia.

Received: 10 February 2020 Accepted: 24 March 2020

Published online: 04 May 2020

\section{References}

1. Steel Z, Marnane C, Iranpour C, Chey T, Jackson JW, Patel V, et al. The global prevalence of common mental disorders: a systematic review and metaanalysis 1980-2013. Int J Epidemiol. 2014;43(2):476-93.

2. Walker ER, McGee RE, Druss BG. Mortality in mental disorders and global disease burden implications: a systematic review and meta-analysis. JAMA Psychiatry. 2015;72(4):334-41.

3. International Diabetes Federation. IDF Diabetes atlas (9th ed). www. diabetesatlas.org/: International Diabetes Federation; 2019.

4. American Diabetes Association. Diagnosis and classification of diabetes mellitus. Diabetes Care. 2011;34(Suppl 1):60-71.
5. de Jonge P, Alonso J, Stein DJ, Kiejna A, Aguilar-Gaxiola S, Viana MC, et al. Associations between DSM-IV mental disorders and diabetes mellitus: a role for impulse control disorders and depression. Diabetologia. 2014;57(4):699-709.

6. Mezuk B, Eaton WW, Albrecht S, Golden SH. Depression and type 2 diabetes over the lifespan: a meta-analysis. Diabetes Care. 2008;31(12):2383-90.

7. Al-Atram AA. A review of the bidirectional relationship between psychiatric disorders and diabetes mellitus. Neurosciences. 2018;23(2):91-6.

8. De Hert M, Dekker JM, Wood D, Kahl KG, Holt Rl, Moller HJ. Cardiovascular disease and diabetes in people with severe mental illness position statement from the European Psychiatric Association (EPA), supported by the European Association for the Study of Diabetes (EASD) and the European Society of Cardiology (ESC). Eur Psychiatry. 2009;24(6):412-24.

9. McBain H, Lamontagne-Godwin F, Haddad M, Simpson A, Chapman J, Jones J, et al. Management of type 2 diabetes mellitus in people with severe mental illness: an online cross-sectional survey of healthcare professionals. BMJ Open. 2018;8(2):e019400.

10. Jørgensen M, Mainz J, Carinci F, Thomsen RW, Johnsen SP. Quality and Predictors of Diabetes Care Among Patients With Schizophrenia: A Danish Nationwide Study. Psychiatr Serv. 2018:69(2):179-85.

11. Stubbs B, Vancampfort D, De Hert M, Mitchell AJ. The prevalence and predictors of type two diabetes mellitus in people with schizophrenia: a systematic review and comparative meta-analysis. Acta Psychiatr Scand. 2015;132(2):144-57.

12. Vancampfort D, Mitchell AJ, De Hert M, Sienaert P, Probst M, Buys R, et al. Type 2 diabetes in patients with major depressive disorder: a meta-analysis of prevalence estimates and predictors. Depress anxiety. 2015;32(10):763-73.

13. Vancampfort D, Mitchell AJ, De Hert M, Sienaert P, Probst M, Buys R, et al. Prevalence and predictors of type 2 diabetes mellitus in people with bipolar disorder: a systematic review and meta-analysis. J Clin Psychiatry. 2015; 76(11):1490-9

14. Vancampfort D, Rosenbaum S, Ward PB, Steel Z, Lederman O, Lamwaka AV, et al. Type 2 diabetes among people with posttraumatic stress disorder: systematic review and meta-analysis. Psychosom Med. 2016;78(4):465-73.

15. Shamseer L, Moher D, Clarke M, Ghersi D, Liberati A, Petticrew M, et al. Preferred reporting items for systematic review and meta-analysis protocols (PRISMA-P) 2015: elaboration and explanation. BMJ. 2015;349:g7647.

16. American Psychiatric Association. Diagnostic and statistical manual of mental disorders: DSM-III. Washington, DC: American Psychiatric Association; 1980.

17. American Psychiatric Association. Diagnostic and statistical manual of mental disorders: DSM-IV-TR. Washington, DC: American Psychiatric Association; 2000

18. American Psychiatric Association. Diagnostic and statistical manual of mental disorders (5th ed.). Arlington, VA: American Psychiatric Association;2013.

19. World Health Organization. The ICD-10 classification of mental and behavioural disorders: clinical descriptions and diagnostic guidelines. Geneva: World Health Organization; 2009.

20. Covidence [Computer program]. Covidence. Melbourne, Australia: Veritas Health Innovation. Available at www.covidence.org; accessed 1 June 2018.

21. Barendregt JJ, Doi SA, Lee YY, Norman RE, Vos T. Meta-analysis of prevalence. J Epidemiol Community Health. 2013;67(11):974-8.

22. Pollock M, Fernandes RM, Becker LA, Pieper D, Hartling L. Chapter V: Overviews of Reviews. In: Higgins JPT, Thomas J, Chandler J, Cumpston M, Li T, Page MJ, Welch VA (editors). Cochrane Handbook for Systematic Reviews of Interventions version 6.0 (updated March 2020). Cochrane, 2020.

23. Cochran WG. The combination of estimates from different experiments. Biometrics. 1954;10:101-29.

24. Higgins JP, Thompson SG. Quantifying heterogeneity in a meta-analysis. Stat Med. 2002;21(11):1539-58.

25. Egger M, Davey Smith G, Schneider M, Minder C. Bias in meta-analysis detected by a simple, graphical test. BMJ. 1997;315:629-34.

26. Whiting P, Savovic J, Higgins JP, Caldwell DM, Reeves BC, Shea B, et al. ROBIS: A new tool to assess risk of bias in systematic reviews was developed. J Clin Epidemiol. 2016;69:225-34.

\section{Publisher's Note}

Springer Nature remains neutral with regard to jurisdictional claims in published maps and institutional affiliations. 